\title{
Audiencia social versus audiencia creativa: caso de estudio Twitter
}

\author{
Luis DELTELL ESCOLAR \\ Universidad Complutense de Madrid \\ ldeltell@ccinf.ucm.es
}

Recibido: 27 de diciembre de 2013

Aceptado: 12 de febrero de 2014

\begin{abstract}
Resumen
Este artículo presenta las conclusiones de una investigación sobre la audiencia social y la audiencia creativa en Twitter en España. El objetivo del proyecto es entender el nuevo comportamiento de la comunidad de usuarios de este espacio de microblogging durante la emisión de contenidos televisivos. Se trata, por tanto, de un estudio del manejo de dos, o más, pantallas simultáneamente (Twitter y Televisión -o cualquier otro dispositivo que permita contemplar los programas de la TV). Se afronta con una metodología dual con técnicas cualitativas y cuantitativas procedentes de diversas áreas de conocimiento y se trata de una propuesta multidisciplinar. Entre los objetivos del proyecto se encuentra mostrar si es posible medir el número total de telespectadores por medio del flujo de tweets y describir cuáles son los líderes del debate de la televisión social.
\end{abstract}

Palabras clave: Twitter, Audiencia Social, Audiencia Creativa, Televisión, blog, microblogging, Internet

\section{Social Audience versus Creative Audience: Case Study Twitter}

\begin{abstract}
This paper presents the conclusions of research over social and creative audiences on Twitter in Spain. The goal of the project is to understand the new way the user community of this microblogging space behave during TV broadcasts. It is, therefore, a study of the use of two or more screens simultaneously (Twitter and Television -or any other device to watch TV shows). The research is conducted through dual methodology, using both qualitative and quantitative techniques from different areas of knowledge, hence being a multidisciplinary proposal. Among the goals of the project lies to prove whether it is possible to measure the total amount of viewers given the tweet flow and describe the thought leaders within the social TV talk.
\end{abstract}

Keywords: Twitter, Social Audience, Creative Audience, Television, blog, microblogging, Internet

\section{Referencia normalizada}

DELTELL ESCOLAR, Luis (2014): “Audiencia social versus audiencia creativa: caso de estudio Twitter". Estudios sobre el Mensaje Periodístico. Vol. 20, Núm. 1 (enero-junio), págs.: 33-47. Madrid, Servicio de Publicaciones de la Universidad Complutense.

Sumario: 1.Introducción y estado de la cuestión. 2. Metodología y material. 3. Resultados y análisis. 4. Conclusiones. 5. Referencias bibliográficas. 6. Apoyos y agradecimientos.

\section{Introducción y estado de la cuestión}

El espacio de microblogging Twitter se ha convertido rápidamente en una de las web capitales del intercambio y de la creación de opinión en Internet. Su diseño y su funcionamiento se sitúan a medio camino entre los blogs y las redes sociales. Sus breves mensajes, llamados tweets, de menos de 140 caracteres se comportan, a veces, como arteras armas en los debates en la Red. Su éxito fue casi inmediato y el impacto que ha ejercido en la sociedad global es incuestionable. La huella de Twitter se encuentra en las primaveras árabes (Al-Kandari, y Hasanen, 2012; Papacharissi, y Oliveira, 2012), la creación de nuevos líderes y dirigentes que ha recorrido Latinoamérica (Said 
Hung y Arcila Calderón, 2011; González, Mendoza y Petersen, 2010) y la formación de grupos de indignados en Norteamérica -el conocido como Occupy Wall Street(Thorson et al., 2013) y en España -los movimientos del 15M- (Osteso et al.., 2013). Por ello no extraña que los más entusiastas de dicha compañía hayan propuesto, hasta en dos ocasiones, la candidatura de la empresa privada Twitter a los Premios Nobel de la Paz (Morozov, 2009).

Todos los autores e investigadores que se han reflexionado sobre este espacio de microblogging han descubierto dos características esenciales: la inmediatez y la creación de debate. A diferencia de Facebook o Tuenti, que se consolidan como intercambio de afectos y vida cotidiana, Twitter opera, sobre todo, como motor de opinión de manera instantánea. En el mismo momento que sucede un acontecimiento, los usuarios de esta web lanzan sus mensajes, se contestan y se responden. No sorprende que este punto de encuentro haya despertado el interés de los analistas del periodismo (Lasorsa y Holton, 2011), de la opinión pública mundial (Twiplomacy) y de los estudiosos de las campañas políticas (Aparaschivei, 2011; Deltell, Claes y Osteso, 2013). Como exponen Eun$\mathrm{Ju}$ y Yun parece que los dirigentes que utilizan esta web se benefician de una nueva comunicación; más rica, dinámica y emotiva (Eun-Ju y Yun, 2012).

Uno de los aspectos más atractivos de Twitter es su uso como segunda pantalla. Desde que la empresa estadounidense publica sus datos y permite el acceso a parte de su código fuente y el uso de su API (Application Programming Interface) los investigadores han descubierto que el mayor volumen mundial de tweets es causado siempre por una retransmisión televisiva. Las cifras que presenta la propia compañía de California no pueden ser más esclarecedoras. Así, el momento de mayor crecimiento en el flujo de tweets por segundo registrado nunca se produjo con relación a la emisión el 2 de agosto de 2013 de la película El castillo en el cielo (Tenkū no Shiro Rapyuta, 1986 ) de Hayao Miyazaki en Japón; el siguiente instante con mayor actividad de escritura fue durante la emisión en directo del partido de balompié entre el F.C. Barcelona y el Chelsea el 24 de abril de 2013 y el tercero record en el torrente de mensaje se alcanzó durante la Superbowl del 2013. Es decir, los tres picos de actividad están relacionados con contenidos televisivos (datos obtenidos de blog.twitter.com, órgano oficial de la compañía).

Estas cifras demuestran hasta como los programas televisados influyen en Twitter. Pero también presentan un nuevo rol en el espectador. Revelan cómo los espectadores/internautas están modificando sus hábitos de consumo/uso. Ahora, la retrasmisión de un evento deportivo, de una película o de un capítulo de una serie de ficción es percibida por los telespectadores como un objeto opinable y estos se transforman en el tema central de Twitter. Además, la actividad en el contexto mundial, como se observa, no es numéricamente desdeñable sino que refleja una cantidad inmensa de espectadores/internautas que simultanean los dos consumos y usos (TV + Twitter). A estos nuevos espectadores se les denomina, de forma general, con el término audiencia social y, a veces, audiencia creativa (Castells, 2011).

Este artículo resume las conclusiones de una investigación que aborda las diferencias entre la audiencia social, la audiencia creativa y la audiencia tradicional (o audimétrica). De forma sintética para iniciar el trabajo deberíamos entender: audiencia 
social como aquella que utiliza simultáneamente dos pantallas o dispositivos (en nuestro caso de estudio Twitter y la televisión -o cualquier otro aparato desde el cual contempla un contenido televisivo); y audiencia creativa aquella parte de la audiencia social que interactúa y crea opinión (es decir, los espectadores/internautas que no solo escriben en Twitter, sino que logran con sus tweets modificar el discurso sobre el contenido emitido).

No menos interesante es saber si Twitter puede servir para calcular la audiencia real de un programa. Es decir, no solo para contabilizar el número de tweets escritos durante una emisión televisiva sino, también, para prever el número total de telespectadores, como se estima con los sistemas de medición basados en audímetros -audiencia audimétrica. Varias investigaciones y empresas de comunicación han planteado diversas metodologías y prototipos de cálculo del número de espectadores según la actividad que se genera en el espacio de microblogging. Así, la compañía de los EE.UU. Nielsen presentó un modelo (aunque no facilita la metodología del mismo), asimismo se puede consultar el trabajo de la empresa Tuitele y, desde el campo de la investigación, se han publicado varios estudios científicos en Chile (Rojo Pizarro, 2012), España (Claes, Deltell y Osteso, 2013; Congosto et al., 2013) y en el escenario europeo (Highfield, T., Harrington y Bruns, 2013). Además, nuestra investigación indaga en la relación entre audiencia tradicional y audiencias social/creativa.

El eje central de este trabajo es descubrir y entender cuál es la verdadera audiencia creativa que utiliza Twitter dentro de un nuevo modelo de comunicación. En este paradigma el espectador se debe entender como un receptor/emisor. En sintonía con los ciberoptimistas y, especialmente con Manuel Castells, comprendemos que ha comenzado a surgir una nueva audiencia que interactúa con los contenidos audiovisuales de una televisión (o cualquier medio de masas) y logra crear un debate original al respecto. Estos nuevos espectadores no son el grueso o la mayoría de la audiencia social, sino solo una pequeña parte de aquella, la cual no solamente escribe tweets, sino que consigue modificar la dirección de la opinión y dar un nuevo sentido a los programas y eventos retransmitidos.

Esta investigación se centra en un estudio de la audiencia social versus audiencia creativa desde una perspectiva mesurada y mensurada. Es decir, primero desde una visión no ciberoptimista de las herramientas de Internet y de los efectos de Twitter pero tampoco desde una perspectiva desilusionada; y segundo monitorizando el flujo de tweets y las audiencias tradicionales para comparar dichos datos. No menos importante es la perspectiva cualitativa para entender qué es y cómo se comporta un líder de opinión en la Red. Nuestro marco de estudio Twitter y las relaciones sociales, como se ha observado, es un campo reciente pero frenético. En la actualidad sobre este espacio de microblogging se generan un elevado número de investigaciones científicas $\mathrm{y}$, por ello, las conclusiones que presentamos deben situarse en un contexto de cambio y evolución permanente.

\section{Metodología y material}

Nuestra investigación aborda el fenómeno de las nuevas audiencias, aquellos espectadores que utilizan/consumen, a la vez, dos pantallas. Pero se trata de una investiga- 
ción muy precisa que se centra únicamente en el uso de dos pantallas concretas: programas televisivos emitidos y Twitter, es decir, únicamente abordamos el comportamiento de los telespectadores que actúan en este espacio de microblogging.

Este estudio plantea tres objetivos de trabajo:

A. Análisis de audiencia audimétrica y audiencia social/creativa. Se muestra los modelos de medición del número de espectadores por medio del flujo de tweets y si existe una correlación entre ambas.

B. Diferencias entre audiencia social y audiencia creativa. Descubrir las desemejanzas en sendos comportamiento y explicar por qué no se deben entender como una única tipología de espectadores/internautas.

C. Estudio y modelo de nuevos patrones de espectadores/internautas dentro del paradigma de la autocomunicación de masas, es decir, plantear casos de audiencia creativa plena.

Para realizar nuestra investigación proponemos una metodología dual: cuantitativa y cualitativa. Así algunas de los objetivos de trabajo requieren de una monitorización detallada mientras otras deben enfocarse desde el análisis comparado y cualitativo. Por ello, recurrimos a herramientas y técnicas de diversas ciencias y perspectivas. No obstante, esta metodología doble no debe ser entendida como segmentada y arbitraria, sino complementaria; que se construye desde diversos campos del saber y de la investigación, por ello, se trata de una técnica multidisciplinar (o más exactamente transdisciplinary).

Para atender el estudio de audiencia audimétrica y audiencia social/creativa se requiere de una monitorización diaria tanto del número de espectadores medidos por audímetros como del volumen de tweets generados por la audiencia social. Para ello recurrimos al estudio de la empresa española GECA, coordinada por Kantar-Media, y a diversas herramientas en red que miden la actividad en Internet: Tuitele y T-hoarder.

La consultoría GECA, Grupo de Estudios de la Comunicación Audiovisual, es la referencia en el sector audiovisual en España. Sus memoranda basados en el parque de audímetros de Kantar-Media no computan la audiencia social, sino el número de telespectadores totales. Estos datos son la referencia básica para crear la medición oficial, es decir, el estudio de GECA sirva para fijar el precio de los anuncios en España.

Para obtener la audiencia social se recurre a Tuitele y T-hoarder: Tuitele es una compañía catalana especializada en la audiencia social de Twitter dentro del contexto español. Dicha empresa facilita en horario de máxima audiencia (prime time) el número de tweets que se escriben en relación con los programas se emiten en ese momento. Además publica diariamente la audiencia social del día anterior. Es a día de hoy, el único barómetro para el cálculo de audiencia social de Twitter en España. El sistema T-hoarder es un modelo académico y científico creado por la investigadora Mari Luz Congosto de la Universidad Carlos III de Madrid. Aunque esta herramienta no se dedica únicamente a la medición audiencia permite comprobar y testar errores de Tuitele. Ambos sistemas de medición se basan en el código API (Application Programming Interface) de Twitter y según una reciente publicación sus resultados son aceptablemente similares y precisos (Congosto et al., 2013). 
En este proyecto se realizan una comparación y una monitorización durante 7 días de la audiencia audimétrica (medida por GECA) y de la audiencia social (medida por Tuitele). Con los datos obtenidos se espera refutar la hipótesis inicial. En la tabla 1 ofrecemos los datos básicos del experimento. La elección de la semana de muestra no es casual, se ha buscado que se tratara de un periodo vacacional en el cual no ocurriese ningún evento televisivo importante. Como es lógico suponer en España el mes de agosto ha sido el escogido, aunque en los días de estudios no han ocurrido acontecimientos políticos y sociales que pudieran hipertrofiar el experimento, sí se han mantenido las competiciones deportivas (algo imposible de eludir en cualquier estudio dado el amplísimo y continuo calendario de las mismas).

Tabla1. Datos básicos del experimento. Fuente: Elaboración propia

\begin{tabular}{|c|c|}
\hline \multicolumn{2}{|r|}{ Datos básicos del experimento } \\
\hline Universo & $\begin{array}{l}\text { España, territorio peninsular, islas Canarias, islas Baleares y ciudades } \\
\text { autónomas de Ceuta y Melilla. }\end{array}$ \\
\hline $\begin{array}{l}\text { Medición } \\
\text { audiencia social } \\
\text { creativa }\end{array}$ & www tuitele.com, T-hoarder \\
\hline $\begin{array}{l}\text { Medición } \\
\text { audiencia } \\
\text { audimétrica }\end{array}$ & www formulatv com, GECA, \\
\hline $\begin{array}{l}\text { Fecha del } \\
\text { Experimento }\end{array}$ & $\begin{array}{l}18 / 08 / 2013,19 / 08 / 2013,20 / 08 / 2013,21 / 08 / 2013,22 / 08 / 2013,23 / 08 / 2013, \\
24 / 08 / 2013\end{array}$ \\
\hline $\begin{array}{l}\text { Herramientas en } \\
\text { Red utilizadas }\end{array}$ & $\begin{array}{l}\text { www.klout.com, www hootsuite.com, www followthehashtag.com, } \\
\text { www retweetrank.com, }\end{array}$ \\
\hline $\begin{array}{l}\text { Consultoras en } \\
\text { Red utilizadas }\end{array}$ & Reputation Metrics, Conectan \\
\hline Control de datos & $\begin{array}{l}\text { Diario. } \\
\text { Minuto a minuto periódico }\end{array}$ \\
\hline $\begin{array}{l}\text { Total de la } \\
\text { muestra }\end{array}$ & $\begin{array}{l}\text { Tweets analizados total: }+/-2.330 .000 \text { mensajes de audiencia social. } \\
\text { Datos entre los cinco programas diarios de más audiencia: } \\
\text { Tweets acumulados: } 1.759 .519 \text {. } \\
\text { Espectadores sociales acumulados: } 857.359 \text {. } \\
\text { Telespectadores acumulados: } 75.152 .000 \text {. }\end{array}$ \\
\hline
\end{tabular}

Para responder a las diferencias entre audiencia social y audiencia creativa se plantea una metodología doble: cuantitativa y cualitativa. Por un lado se recurre a sistemas de monitorización en red: factor Klout, Hashtracking, Hootsuite y resultados de consultorías (como Conectan, Reputation Metrics). El factor Klout, como observa J. Serrano Puche, es junto a PeerIndex la herramienta más valiosa para saber el impacto de los perfiles en Twitter y Facebook (Serrano-Puche, 2012). Hashtracking posibilita el seguimiento de los hashtags más importantes y de los perfiles más implicados en los debates. Por último, Hootsuite facilita una monitorización de los perfiles estudiados. Los memoranda de las consultorías Conectan (de carácter empresarial) y Reputation Metrics (de la Universidad de Navarra en España) nos permiten contrastar resultados.

Se descarta la utilización de cualquier texto o estudio cuya metodología de trabajo no recurra al seguimiento API de Twitter o a herramientas de monitorización propias. Actualmente en el ámbito Latinoamericano e ibérico han surgido diversos consultarías y grupos de investigación sobre Twitter que realizan memorias sobre influencia e impacto pero no recurren nunca al Application Programming Interface de la empresa norteamericana ni presentan metodologías científica y rigurosas. Estos trabajos aunque muy populares, y bien posicionados, en la Red son inciertos en sus resultados.

El estudio y modelo de nuevos ejemplos de espectadores/internautas dentro del modelo de la autocomunicación de masas se debe abordar desde el análisis cualitativo. Para ello seguimos a los usuarios más influentes y se analiza minuciosamente su comportamiento. Citamos los modelos presentados por Castells (Castells, 2011; Castells, 2012) y la propuestas realizadas por A. Marwick (Marwick, 2010; Marwick y Boyd, 2011). 


\section{Resultados y análisis}

Una de las preguntas básicas de este trabajo se refiere a la capacidad de calcular la audiencia global, el número total de telespectadores, con sistemas de medición de la audiencia social por medio de Twitter. El primer objetivo es investigar los prototipos de estimación de la audiencia tradicional a través del seguimiento del caudal de tweets. Se pretende descubrir una relación directa entre el número de mensajes escritos en el espacio de microblogging y los telespectadores totales.

En la actualidad existen varios experimentos científicos sobre esta posible relación entre número de Tweets y espectadores totales. La empresa estadounidense Nielsen ha publicado en varias ocasiones en la prensa generalista que trabaja en modelos de medición, pero ninguno de ellos se ha concretado de forma científica ni se ha presentado a revistas de investigación. También existen trabajos académicos que encuentran una "relación" entre número total diario de tweets y la audiencia de un programa (Rojo, 2012), mas este estudio no realiza un seguimiento del minuto a minuto (básico en las mediciones de audiencia) ni el comportamiento de todos los productos de esa franja horaria ni, siquiera, la actividad de la cadena, por dicho motivo se descarta como posible metodología.

Para realizar nuestro trabajo proponemos una medición semanal (18 de agosto al 24 de agosto de 2013) de todos los programas de la parrilla televisiva española y con la totalidad de los canales disponibles tanto en abierto como en cerrado. Es decir, nos basamos en los criterios universales aplicados por GECA y Kantar-media para la medición en España. Además, recurrimos a un seguimiento monitorizado de los diez programas más vistos de cada día. En los cuales mensuramos la audiencia social en Twitter y la audiencia audimétrica tradicional. Recurrimos a los siguientes parámetros de estudio: espectadores social, tweets totales generados, números de tweets por minuto (se incluyen retweets, menciones, respuestas), número de telespectadores totales, share tradicional en su franja horaria. Estos datos se contabilizan diariamente en los diez programas de más impacto - Tabla 2:

Como se observa en la tabla 2, aunque puede surgir alguna coincidencia -como el caso de la retransmisión del partido entre

Tabla2. Comportamiento de los diez programas más vistos en la audiencia social y en la audiencia audimétrica, ejemplo día 21 de agosto de 2013. Fuente: elaboración propia

\begin{tabular}{|c|c|c|c|c|c|c|c|}
\hline \multirow{2}{*}{$\begin{array}{c}\text { Fecha } \\
2013 \\
21 \text { agosto }\end{array}$} & \multicolumn{4}{|c|}{$\begin{array}{c}\text { Audiencia social } \\
\text { Tuitele }\end{array}$} & \multicolumn{3}{|c|}{$\begin{array}{c}\text { Audiencia audimétrica } \\
\text { GECA, Formulatv.com... }\end{array}$} \\
\hline & Programa & Tweets & Tweets & Espectadores & Programa & Share & Espectadores \\
\hline 1 & $\begin{array}{l}\text { Supercopa } \\
\text { Partido y } \\
\text { previo }\end{array}$ & 308.787 & $1.470,4$ & 132.322 & Supercopa & $38,4 \%$ & 4.930 .000 \\
\hline 2 & $\begin{array}{l}\text { Mujeres y } \\
\text { hombres y } \\
\text { viceversa }\end{array}$ & 62.281 & 377,4 & 36.195 & $\begin{array}{l}\text { Previo: } \\
\text { Supercopa }\end{array}$ & $21,4 \%$ & 2.927 .000 \\
\hline 3 & Punto Pelota & 11.175 & 74,5 & 7.041 & $\begin{array}{l}\text { El secreto } \\
\text { de Puente } \\
\text { Viejo }\end{array}$ & $19,5 \%$ & 1.881 .000 \\
\hline 4 & Baloncesto & 9.985 & 60,5 & 7.293 & Los Simpson & $16,2 \%$ & 1.660 .000 \\
\hline 5 & $\begin{array}{l}\text { Deportes } \\
\text { Cuatro }\end{array}$ & 7.524 & 65,4 & 6.421 & $\begin{array}{l}\text { Antena } 3 \\
\text { Noticias } 1\end{array}$ & $14,8 \%$ & 1.659 .000 \\
\hline 6 & Salvane:Diario & 2.491 & 7,9 & 1.297 & $\begin{array}{l}\text { Pasapalabra } \\
\text { verano }\end{array}$ & $16,9 \%$ & 1.425 .000 \\
\hline 7 & $\begin{array}{l}\text { El video del } \\
\text { verano }\end{array}$ & 2.142 & 14,2 & 1.029 & Telediaro I & $12,5 \%$ & 1.416 .000 \\
\hline 8 & $\begin{array}{l}\text { Energy Tiki- } \\
\text { taka }\end{array}$ & 1.712 & 10,4 & 1.198 & $\begin{array}{l}\text { Informativos } \\
\text { Telecinco } \\
15: 00\end{array}$ & $12,8 \%$ & 1.409 .000 \\
\hline 9 & $\begin{array}{l}\text { De hoy a } \\
\text { mañana }\end{array}$ & 1.412 & 6,5 & 655 & $\begin{array}{l}\text { Amar es } \\
\text { para } \\
\text { siempre }\end{array}$ & $14,4 \%$ & 1.399 .000 \\
\hline 10 & $\begin{array}{l}\text { Hay una cosa } \\
\text { que te quiero } \\
\text { decir }\end{array}$ & 1.238 & 3,9 & 1.031 & $\begin{array}{l}\text { Informativos } \\
\text { Telecinco }\end{array}$ & $12,8 \%$ & 1.333 .000 \\
\hline \multirow[t]{2}{*}{$\frac{1}{\text { TOTALES }}$} & & Tweets & Media & Espectadores & & & Espectadores \\
\hline & & 407.509 & 20,87 & 193.451 & & & 18.706 .000 \\
\hline
\end{tabular}


el Atlético de Madrid y Barcelona F.C.-, lo cierto es que no hay una correlación continua. La audiencia social y la audiencia audimétrica tienen un comportamiento y hábito diverso y es imposible medir la una con los datos de la otra. Se trata de un modo de consumo diferente y, aún más, extraño entre ambos.

Un dato importante como ya se observó, (Claes, Deltell y Osteso, 2013), son las unidades y cantidades medidas. En el caso de la audiencia social es irrisorio el volumen de tweets: los programas más vistos (en especial de los 6 al 10) ofrecen una media de menos de 3.000 tweets por producto y una actividad de 5,8 tweets por minuto. Absolutamente alejado de las audiencias audimétricas con un volumen de millones de espectadores.

Para mostrar con más claridad el comportamiento extraño que se produce entre ambas audiencias se sitúa en la tabla 3 el seguimiento de los cinco programas más vistos diariamente durante el periodo del experimento. Se observa como las coincidencias son mínimas y hay que hablar de una audiencia distinta y de un profundo extrañamiento entre ambas comunidades de público.

La tabla 3 esclarece el extrañamiento entre ambas audiencias. Ninguna de las jornadas estudias permitió establecer una relación directa entre el comportamiento del movimiento de tweets y la audiencia de los programas o la actividad de las parrillas de programación televisiva española.

Tabla3. Medición de los cinco programas de más audiencias (social y audimétrica) desde el 18 de agosto al 24 de agosto año 2013.

Fuente: elaboración propia

\begin{tabular}{|c|c|c|c|c|c|c|}
\hline & & & & & $\begin{array}{c}\text { Audienci } \\
\text { Audimárica }\end{array}$ & \\
\hline ago Canal & Programa & Tweets & Espectadores & Canal & Programa & Audiencis \\
\hline 1 Canal + & $\begin{array}{l}\text { Real M } \\
\text { Betis }\end{array}$ & 167.909 & 75.719 & Antena3 & E1 peliculon & 2.064 .000 \\
\hline 2 Canal + & $\begin{array}{l}\text { Barcelor } \\
\text { Levante }\end{array}$ & 105.032 & 51.029 & La1 & Peliabla semana & 2.039 .000 \\
\hline 3 Antena 3 & E1 peliculón & 53.915 & 35269 & Antena3 & Multicine & 1.787 .000 \\
\hline $\begin{array}{l}4 \text { Telecinco } \\
5 \text { La1 }\end{array}$ & $\begin{array}{l}\text { Mindial } \\
\text { motociclismo } \\
\text { Master } 1000 \\
\text { Cincimati }\end{array}$ & $\begin{array}{l}29.625 \\
28.484\end{array}$ & $\begin{array}{l}13948 \\
18370 \\
\end{array}$ & Antena3 & $\begin{array}{l}\text { Mulicine 2 } \\
\text { Tenis:Misters } \\
1000\end{array}$ & $\begin{array}{l}1.773 .000 \\
1.575 .000\end{array}$ \\
\hline $\begin{array}{l}\text { 19- } \\
\text { ago }\end{array}$ & Frograr & Tweets & Espectadores & Canal & Programa & Audiencia \\
\hline 1 Telecinco & $\begin{array}{l}\text { Mijeres y } \\
\text { hombres y } \\
\text { viceversa }\end{array}$ & 46.980 & 29.835 & Antena3 & $\begin{array}{l}\text { E1 screto de } \\
\text { Puent Viejo }\end{array}$ & 1.884 .000 \\
\hline 2 Cuatro & Celta-Espanyol & 30.057 & 15.164 & Antena3 & Los Simpson & 1.745 .000 \\
\hline 3 Intereconomia & PuntoPelota & 19.216 & 10518 & Telecinco & Sálvame:Diario & 1.713 .000 \\
\hline 4 Cuatro & Tiki-Taka & 15.293 & 10.062 & Lal & Telediariol & 1.649 .000 \\
\hline 5 Telecinco & $\begin{array}{l}\text { Campamento de } \\
\text { verano }\end{array}$ & 10.532 & 4.454 & Antena3 & Ioticias & 16.300 .000 \\
\hline ago Canal & Programa & Tweets & Espectadores & Canal & Programa & Audiencis \\
\hline 1 La1/TV3 & $\begin{array}{l}\text { Champions } \\
\text { League } \\
\text { Myjeres y } \\
\text { hombres y } \\
\text { viceversi }\end{array}$ & 78.831 & 42330 & Antena3 & ChampionsLeague & 2.181 .000 \\
\hline 3 Intereconomia & \begin{tabular}{|l|l|} 
PuntoPelota \\
\end{tabular} & 19.938 & 9.769 & Lal & & 1.954 .000 \\
\hline 4 Teledeporte & $\begin{array}{l}\text { Balonce: } \\
\text { Mac }\end{array}$ & 8.931 & 4602 & Antena3 & Los Simpson & 1.941 .000 \\
\hline LaSexta3 & $\begin{array}{l}\text { Los chicos del } \\
\text { coro }\end{array}$ & 6.673 & 5.444 & Antena3 & $\begin{array}{l}\text { Los secetos de } \\
\text { Puent Viejo }\end{array}$ & 1.828 .000 \\
\hline ago Canal & Programa & Twrets & Espectadores & Canal & Programa & Audiencia \\
\hline 1 La 1 & Supercopa & 308.787 & 132322 & Lal & Superopa & 4.930 .000 \\
\hline $\begin{array}{ll}2 & \text { Telecinco } \\
3 & \text { Intereconomin }\end{array}$ & $\begin{array}{l}\text { Mijerese y } \\
\text { hombres y } \\
\text { viceversa } \\
\text { PuntoPelota }\end{array}$ & $\begin{array}{l}62.281 \\
11.175\end{array}$ & 36.195 & \begin{tabular}{|l} 
La1 \\
Antena33
\end{tabular} & $\begin{array}{l}\text { Previo: Supercopa } \\
\text { El sercetode } \\
\text { Puent Viejo }\end{array}$ & 2.927 .000 \\
\hline 4 Teledeporte & Baloncesto & 9.985 & 7293 & Antena3 & Los Simpson & 1.660 .000 \\
\hline 5 Cuatro & Depottes & 7.524 & 6.421 & Antena3 & Noticias 1 & 1.659 .000 \\
\hline $\begin{array}{l}22- \\
\text { ago Canal }\end{array}$ & Progra: & Twreets] & Espectadores & Canal & Programa & Audiencia \\
\hline 1 Antera3 & $\begin{array}{l}\text { Trofeo Santiago } \\
\text { Bernabeú }\end{array}$ & 289.535 & 122798 & Antena3 & Bernabéu & 3.479 .000 \\
\hline 2 Telecinco & $\begin{array}{l}\text { Mijeres y } \\
\text { hombres y } \\
\text { viceversa }\end{array}$ & 197.911 & 87.035 & Antena3 & feoco & 2.903 .000 \\
\hline 3 Telecinco & $\begin{array}{l}\text { Campamento de } \\
\text { verano }\end{array}$ & 23.467| & 8.826 & Antena3 & $\begin{array}{l}\text { E1 secreto de } \\
\text { Puente Viejo }\end{array}$ & 1.810 .000 \\
\hline 4 Intereconomia & PuntoPelota & 20.392 & 10343 & Antena3 & Los Simpson & 1.699 .000 \\
\hline 5 Cuatro & Deportes & 3.682 & 3255 & Telecinco & Salvame:Diario & 1.687 .000 \\
\hline $\begin{array}{l}23- \\
\text { ago } \\
\text { agnal }\end{array}$ & Prograr & Tweets & Eppectadores & Canal & ama & Audiencia \\
\hline 1 Telecinco & $\begin{array}{l}\begin{array}{l}\text { Mijeres y } \\
\text { hombres y } \\
\text { vicevers }\end{array} \\
\end{array}$ & 83.689 & 45252 & Antena3 & & 1.790 .000 \\
\hline 2 Cuatro & $\begin{array}{l}\text { Fubol:Atiletic- } \\
\text { Osasuna }\end{array}$ & 29.231 & 13203 & Antena3 & Noticias 1 & 1.632 .000 \\
\hline 3 Telecinco & E1 Dehxe & 12.627 & 4370 & Antena3 & Los Simpson & 1.605 .000 \\
\hline 4 La1 & Baloncesto & 11.626 & 5.899 & Telecinco & Sálvame:Diario & 1.579 .000 \\
\hline 5 Nitro & Fórmula 1 & 6.923 & 4.169 & Telecinco & Informativos & 1.455 .000 \\
\hline $\begin{array}{l}24 . \\
\text { ago Canal }\end{array}$ & Programa & Tweets & Espectadores & Canal & Programa & Audiencia \\
\hline 1 Antena 3 & Formula 1 & 30.384 & 14.677 & Antena3 & Fórmula 1 & 1.855 .000 \\
\hline 2 LaSexta & LaSexta Noche & 23.361 & 8.030 & Lal & Telediario & 1586.000 \\
\hline 3 Telecinco & & 14.337 & 8282 & Antena3 & Multicine & 1.524 .000 \\
\hline 4) Telecinco & \begin{tabular}{|l} 
Mundial \\
motociclismo
\end{tabular} & 7.953 & 3.728 & Antena3 & Noticias & 1.499 .000 \\
\hline 5 La1 & $\begin{array}{l}\text { Ciclismo:La } \\
\text { Vuelta }\end{array}$ & 7.315 & 4590 & Lal & Sesión de tarde & 1354.000 \\
\hline
\end{tabular}


Un caso elocuente del comportamiento de la audiencia social es el poco interés que despierta en esta comunidad virtual algunas series de televisión (nos referimos a Los Simpson y a El secreto de Puente Viejo). Aún menos participativos se muestran los usuarios de Twitter en relación a los informativos.

Los resultados del primer experimento nos muestran que no se puede, en el entorno geográfico y social de España, utilizar hoy el flujo de actividad en Twitter para medir la audiencia audimétrica.

Si bien resulta fundamental mostrar que la audiencia social en Twitter no revela el volumen del número total de telespectadores, también es importante explicar con claridad la diferencia entre audiencia social y audiencia creativa. Estos conceptos llevan a equívocos graves ya que se usan aleatoriamente en la prensa generalista. Como planteamos en nuestro segundo objetivo de la investigación: pretendemos dibujar las diferencias de comportamiento entre la actividad social y la actividad creativa y lo que esto implica en la tipología espectadores/internautas.

En la literatura científica existen diversos trabajos precedentes que nos servir de base para nuestro experimento. El primero de ellos refleja el comportamiento en Twitter del hashtag sobre Eurovisión 2012 (Highfield, T., 2013). Este estudio ideado desde Australia refleja cuál fue el devenir de los internautas durante la emisión del célebre concurso de canciones europeo. Sin duda, este artículo plasma como un evento televisado se puede convertir en un "fenómeno global". Algo parecido, aunque más modesto en sus dimensiones geográficas, es el estudio sobre la retransmisión de los Premios Goya 2013 en España (Congosto et al., 2013). Ambas investigaciones aunque valiosas reflejan una hipertrofia ya que analizan el comportamiento en Twitter en sendos grandes eventos. Es decir, investigan el cauce de tweets en el instante de mayor actividad y, por eso, sus resultados son deslumbrantes.

Todo lo contrario nuestro estudio muestra a la audiencia social en una semana sin eventos mayúsculos (salvo competiciones deportivas). Así nuestro primer resultado es, la evidencia, de una baja participación de la audiencia social en Twitter, mucho menor de la percibida en la transmisión de grandes acontecimientos. Nuestro experimento refleja que los nuevos espectadores son menos de los que se observan en las estimaciones más generosas (Prádanos, 2012).

Para entender la diferencia entre audiencia social y audiencia tradicional se debe observar cuál fue la actividad y la tendencia marcada por los nativos digitales. Como sostiene Prensky: los nativos digitales reflejan los comportamiento más avanzados en la Red (Prensky, 2001). Sin embargo, lo que se observa es lo contrario. Los programas dirigidos a nativos digitales no tienen un gran impacto en la audiencia social. El caso de Los Simpson es especialmente significativo con una audiencia estable 1.600 .000 de telespectadores de los cuales el 45\% (datos GECA) son nativos digitales no alcanza ninguno de los cinco días la centena de tweets. Lo mismo ocurre con los programas para público adolescente de las cadenas de menores: Boing, Clan o Disney Channel.

Los programas como El gato al agua con una audiencia insignificante, de menos de 100.000 espectadores, y con una media de edad superior a los cuarenta años $(50 \%$, datos GECA) logra situarse casi todos los días entre los diez programas más vistos por 
la audiencia social con una media de unos 2.300 tweets. En nuestro experimento, sorprendentemente, la audiencia social, por tanto, es mayoritariamente adulta y no sigue patrones juveniles y adolescentes.

Según Manuel Castells, quien formuló el concepto de audiencia creativa, la diferencia entre la audiencia tradicional y la nueva audiencia era su comportamiento proactivo y discursivo. En este sentido, los comentarios a un programa conservador de debate político, como es El gato al agua, podrían inducirnos a creer que esta audiencia social ejemplifica una intención ideológica. Pero, no es así, ya que tres de los cinco programas con más tweets son siempre de carácter deportivo. Los eventos de fútbol, fórmula 1, ciclismo y baloncesto son lo que más mensajes generan. En ellos los internautas no pretenden crear un debate político o social sino solamente celebrar, como hinchada, los aciertos de sus equipos o de sus deportistas favoritos. Castells insiste que la autocomunicación de masas y el comportamiento creativo debería ser una lucha de poder (Castells, 2011). En todos los programas deportivos y en su actividad de Twitter no se encuentra esta actitud beligerante. Por ello, lo que se desprende de nuestra investigación es que la audiencia social no es plenamente creativa. La mayoría de los comentarios que se generan en Twitter no pretenden crear un debate horizontal o de cuestionamiento de poder y de valor comunicativo, todo lo contrario parecen reafirmar a las instituciones públicas, a los mas media y a los poderes tradicionales.

En este sentido nos resulta interesante mostrar como series como Los Simpson o El secreto de Puente Viejo presentan una gran audiencia audimétrica, con un elevado share. Mientras series como El Barco pioneras en la comunicación transmedia (Grandío y Bonaut 2012). y en el uso de Twitter resultaron un fracaso de audiencia (Fernández 2011). La audiencia social no es necesariamente audiencia creativa. Aquellas personas, que utilizan Twitter mientras contemplan un contenido televisivo no pretenden, en su mayoría, crear un debate o construir una "comunicación horizontal".

Acertadamente los profesores García García y Gértudrix describieron el paradigma digital como un "Mare Nostrum" plagado de "mitos e ideología" (García García, Gértrudix, 2009). Así en nuestro experimento mostramos como, al menos en el ámbito de la muestra, la audiencia social, la audiencia creativa y la audiencia audimétrica son distintas y que no pueden deducirse el uno de la otra. Uno de los "mitos" de la nueva tecnología es la elaboración de calificaciones y listados de liderazgo. Por ello, existen muchos modelos aplicados a Twitter. Sin embargo, estos conllevan con frecuencia errores y contradicciones fundamentales.

El primer aspecto para entender que es un líder de opinión en la audiencia creativa es entender cuál es el carácter o naturaleza de la cuenta estudiada. Existen tres categorías básicas: institucional (aquellos perfiles que representan a una organización existente), personales (aquellas cuentas que son el perfil de un individuo) y los PSR, o personajes surgidos en la red (estos son entidades que han nacido en el propio Twitter o en otras redes sociales). Como indica Castells, la audiencia creativa son personas o individuos alejados de los centros de poder, por lo tanto, las instituciones no pueden ser líderes de audiencia creativa. Lógicamente las organizaciones tradicionales de poder sí pueden ser líderes de la audiencia social y fomentar la participación de sus espectadores pero nunca pueden favorecer la "comunicación horizontal" ya que 
esto implicaría precisamente la pérdida de su poder y de su valía. En nuestro estudio ignoramos todos los perfiles de cadenas de televisión, de cuentas oficiales de programas, periódicos y otros medios tradicionales. Investigamos solo cuentas personales o PSR. Sin embargo, hemos incluido una institucional @El-Hormiguero y una semiinstitucional o semi-personal la del periodista Jordi Évole, que utiliza su cuenta para promocionar su programa televisivo. Estas dos singularidades se deben al comportamiento colaborativo que plantean en sus perfiles.

Los factores que nos permiten hablar de líderes de la audiencia creativa son los siguientes: el número de seguidores en Twitter y su Factor Klout (que como indicamos es el sistema más aceptado por la comunidad científica); la cantidad de retweets máximo logrado durante el periodo de la muestra (es decir, el número total de otros perfiles que han celebrado el mensaje y lo han lanzado a sus comunidades); el volumen de tweets emitidos (un número excesivo de tweets refleja una actividad hipertrófica); su carácter o naturaleza; y su comportamiento si interactúan (Inter.), si ofrecen enlaces (Enl.) y si las cuentas han sido verificadas por Twitter (v.). En la tabla 4 ofrecemos el listado de los 16 líderes observados, ordenada por número total de seguidores (esto no implica un mayor impacto).

Tabla 4. Líderes de la audiencia creativa en el período de la muestra del experimento.

Fuente: elaboración propia

\begin{tabular}{|c|c|c|c|c|c|c|c|c|}
\hline Usuario & Tweets & $\begin{array}{c}\mathrm{Re}- \\
\text { tweets } \\
\text { máximo }\end{array}$ & Seguidores & v. & $\begin{array}{l}\text { Factor } \\
\text { Klout }\end{array}$ & Carácter & Inter. & Enl. \\
\hline @SSegura & 35.707 & 1.154 & 1.829 .550 & No & 73 & Personal & $\overline{\mathrm{Si}}$ & $\mathrm{Si}$ \\
\hline$\underset{\text { @ danimartinezwe }}{\text { a }}$ & 15.553 & 461 & 1.522 .154 & $\mathrm{Si}$ & 72 & $\begin{array}{c}\text { Personal } \\
\text { PSR }\end{array}$ & $\mathrm{Si}$ & $\mathrm{Si}$ \\
\hline @Buenafuente & 10.618 & 256 & 1.501 .161 & $\mathrm{Si}$ & 80 & Personal & No & No \\
\hline @ ristomejide & 9.310 & 4.032 & 1.480 .718 & $\mathrm{Si}$ & 82 & Personal & $\mathrm{Si}$ & $\mathrm{Si}$ \\
\hline @El-Hormiguero & 13.843 & 62 & 1.375 .291 & $\mathrm{Si}$ & 81 & $\begin{array}{c}\text { Institucion } \\
\mathrm{al}\end{array}$ & No & $\cdots$ \\
\hline @AnnaSimonMarí & 1.819 & 67 & 1.139 .037 & $\mathrm{Si}$ & 68 & Personal & No & $\mathrm{Si}$ \\
\hline @CristiPedroche & 4.444 & 68 & 1.109 .173 & $\mathrm{Si}$ & 66 & Personal & $\cdots$ & $\mathrm{Si}$ \\
\hline @jordievole & 7.948 & 254 & 999.037 & $\mathrm{Si}$ & 72 & Personal & $\mathrm{Si}$ & $\cdots$ \\
\hline @pacoleonbarrios & 2.521 & 206 & 976.431 & $\mathrm{Si}$ & 79 & Personal & No & No \\
\hline @berto_romero & 11.962 & 2.040 & 943.320 & $\mathrm{Si}$ & 80 & Personal & Sí & $\cdots$ \\
\hline @.MejoresTwits & 8.218 & 2.351 & 733.408 & No & $\cdots$ & $\begin{array}{c}\text { Humor } \\
\text { PSR }\end{array}$ & No & $\cdots$ \\
\hline @_anapastor & 29.444 & 482 & 718.883 & $\mathrm{Si}$ & 58 & Personal & No & $\ldots$ \\
\hline @Colmenero & 6.130 & 550 & 448.447 & $\mathrm{Si}$ & 72 & $\begin{array}{l}\text { Parodia } \\
\text { PSR }\end{array}$ & $\cdots$ & No \\
\hline @Llourinho & 11.239 & 2.370 & 417.477 & No & 78 & $\begin{array}{l}\text { Parodia } \\
\text { PSR }\end{array}$ & No & $\mathrm{Si}$ \\
\hline @postureo & 2.929 & 600 & 280.775 & No & 72 & $\begin{array}{l}\text { Personal } \\
\text { PSR }\end{array}$ & Si & $\mathrm{Si}$ \\
\hline @hematocritico & 43.8446 & 40 & 32.928 & No & 75 & $\begin{array}{l}\text { Humor } \\
\text { PSR }\end{array}$ & $\mathrm{Si}$ & $\mathrm{Si}$ \\
\hline
\end{tabular}

La monitorización de estos perfiles nos permite descubrir algunos aspectos novedosos. Al tratarse de una semana de baja actividad (tradicionalmente de vacaciones) los periodistas y los medios institucionales no realizan una gran actividad. Así, los perfiles de Ana Pastor, del programa El Hormiguero o de Buenafuente se encuentran menos participativos. Sin embargo, los PSR ofrecen un comportamiento distinto y no descansan en el período vacacional, tanto Llourinho como Postureo, Colmenero escriben, comentan y realizan retweets de forma constante. 
Como se detalla en la tabla 4 todos los perfiles salvo @ hematocritico superan los 250.000 seguidores. Además once de ellos se encuentra cerca o superan el millón. Santiago Segura logra el máximo del estudio con 1.829.550. A pesar de su nivel bajo, @ hematocritico es capaz de situar un hashtag en el entorno nacional sobre casi cualquier tema televisivo e, incluso, en determinados eventos logran ser una tendencia mundial (Congosto et al., 2013). Todos los perfiles muestran una gran actividad con una media de más de 15.000 tweets y, a pesar, de las fechas vacacionales sus tweets tienen impacto en la comunidad virtual. Así cada líder logra al menos que uno de sus mensajes sea retweeteado por más de 60 usuarios, llegando a la cifra de 4.002 retweets obtenida por un texto de Risto Mejide.

Casi la totalidad de los líderes once del total $(68,75 \%)$ recurren al humor y a la parodia para la escritura de sus tweets. Aunque no todos los mensajes emitidos pretenden ser cómicos, es cierto, que el tono general de sus cuentas es la comedia. Esto no implica que los mensajes estén cargados de cometidos, ya que como se observó en el caso de la política de Australia, el humor es un elemento esencial en esta nueva comunicación social (Wilson, 2011).

La mayoría de los usuarios emplean los enlaces y muestra fotografías o imágenes (casi siempre se recurre a plataformas de apoyo como Instragram.com). Muchos de ellos realizan retweeets y comentan los mensajes de otros aunque esto no garantiza un mejor posicionamiento hacerlo. Curiosamente uno de los más proactivos en las menciones, respuestas y comentarios es Santiago Segura responde a la casi totalidad de internautas que le nombran en Twitter. Por último, se observa que las cuentas verificadas no implican un mayor impacto en el liderazgo y así personas, como el citado Santiago Segura, a pesar de no estar verificado por la compañía, presenta el mayor número de seguidores.

Un dato sorprendente y alarmante del estudio de los líderes de opinión es la baja participación de las mujeres en la creación de opinión. De los 16 perfiles estudiados únicamente 3 pertenecen a mujeres. Esto supone el $18,7 \%$ del total una cifra preocupante. No solo se encuentra un número mínimo de perfiles femeninos, sino que además de los tres analizados, únicamente el de Ana Pastor es plenamente creadora de opinión.

\section{Conclusiones}

Este artículo ofrece el resumen de una investigación sobre la audiencia social y la audiencia creativa en Twitter. El eje central del proyecto es entender el nuevo comportamiento de la comunidad de usuarios de este espacio de microblogging durante la emisión de contenidos televisivos. Se trata, por tanto, de un estudio del manejo de dos, o más, pantallas simultáneamente (Twitter y televisión -o cualquier otro dispositivo que permita contemplar los programas de la TV).

Tres son los objetivos de trabajo del estudio:

Primero intentamos descubrir si existe una relación entre audiencia audimétrica y audiencia social y, por lo tanto, si es posible deducir el número total de telespectadores por medio del análisis del flujo de tweets. En nuestra investigación realizamos, durante una semana, la monitorización de todos los mensajes relacionados con la televisión española. Al comparar estos datos con la audiencia audimétrica se demues- 
tra que no hay una relación posible entre ambas. La audiencia social tiene un comportamiento extraño con relación a la audiencia tradicional, no es posible, en el entorno español, deducir el número de telespectadores totales por medio de Twitter. Nuestra investigación muestra que no solo no coinciden los programas más vistos por la audiencia social y la tradicional sino, también, que las cifras de una y otra son de magnitudes no comparables.

Segundo realizamos una cartografía para diferenciar la audiencia social de la audiencia creativa en Twitter. Aunque la prensa generalista utiliza los dos términos como sinónimos lo cierto es que no lo son. La segunda es una comunidad, como indica Castells, que busca una comunicación horizontal y la creación de un debate y una opinión. Se trata de una restructuración del poder. Sin embargo, en nuestro caso de estudio, se percibe que la inmensa actividad de la audiencia social no busca la construcción de un debate o la elaboración de un discurso de valor o poder. Los usuarios del espacio de microblogging evitan los temas discursivos y escriben principalmente sobre eventos deportivos y como hinchadas de equipos y de deportistas.

Tercero, nuestro último objetivo se basa en el estudio de los líderes de la audiencia creativa. Es decir, indagamos cómo son los usuarios que sí crean un debate y logran transmitir opinión sobre los contenidos televisivos. Entre ellos encontramos que la mayoría de los líderes son proactivos, es decir comparten noticias, mencionan, contestan a otros usuarios y realizan retweets. Casi el $70 \%$ de los perfiles utiliza el humor, la irónico o la comedia, sus mensajes suelen ser de carácter lúdico, aunque contengan elementos para la creación de opinión y debate.

Los usuarios con más impacto presentan siempre un factor Klout alto de 60 a 82 puntos. Además, sus cuentas tienen un gran número de seguidores, salvo el caso de (a) hematocritico todos ellos superan los 250.000 seguidores y once se encuentran cerca o sobrepasan el millón. Por último, durante la semana estudiada el conjunto íntegro monitorizado presenta algún mensaje que supera los 60 retweets. Estos tres elementos nos permiten hablar de creadores de opinión en torno a la televisión.

Un dato sorprendente y triste de nuestra investigación es la baja participación de la mujer en el liderazgo de la opinión y del debate en Twitter sobre los contenidos audiovisuales. Las cuentas que pertenecen a mujeres representan menos del $20 \%$ del total de los perfiles con más impacto y prestigio.

\section{Referencias bibliográficas}

AL KANDARI, Ali \& HASANEN, Mohammed (2012): “The impact of the Internet on political attitudes in Kuwait and Egypt". Telematics \& Informatics, 29 (3), 245253. Doi: 10.1016/j.tele.2011.10.005.

APARASCHIVEI, Paul Andrei (2011): "The Use of New Media in Electoral Campaigns: Analysis on the Use of Blogs, Facebook, Twitter and YouTube in the 2009 Romanian Presidential Campaign". Journal of Media Research, 2(10). Cluj-Napoca, Accent Publisher, pp. 39-60.

CASTELLS, Manuel (2011): Comunicación y Poder, Madrid (España), Alianza Editorial. 
CASTELLS, Manuel 2012): Networks of Outrage and Hope: Social Movements in the Internet Age. London, Polity Press.

CLAES, Florencia; OSTESO, José Miguel; y DELTELL, Luis; (2013): “Audiencias televisivas y líderes de opinión en Twitter. Caso de estudio: El Barco". Estudios sobre el Mensaje Periodístico. Vol. 19, núm. 1 (enero-junio), págs. 347-364. Doi: 10.5209/rev_ESMP.2013.v19.n1.42526. Madrid, Servicio de Publicaciones de la Universidad Complutense

CONGOSTO. Mari Luz; DELTELL, Luis; CLAES, Florencia; y OSTESO, José Miguel (2013): "Análisis de la audiencia social por medio de Twitter. Caso de estudio: Los premios Goya 2013". Icono 14. V. 11. N. 2. Julio 52-80. Doi: 10.7195/ri14.v11i2.577.

DELTELL, Luis; CLAES, Florencia; y OSTESO, José Miguel (2013): "Predicción de tendencia política por Twitter: Elecciones Andaluzas 2012". Ámbitos. Revista Internacional de Comunicación, vol. 22, primer semestre.

FERNÁNDEZ MATEOS, Paula (2011): "La televisión social en España: los "twittersodios' de El Barco", en Viernes Digital: diariodepracticasuc3m.com/articulo_imprimir.asp?idarticulo $=28 \&$ accion $=[$ fecha de consulta: 8 de febrero de 2012].

FERRERAS RODRÍGUEZ, Eva María (2011): “La estrategia de la corporación EITB (Euskal Irratia Telebista) en Facebook y Twitter". Razón y Palabra, 76. México D.F., TEC de Monterrey, pp. 1-25.

GARCÍA, Francisco y GÉRTRUDIX, Manuel (2009): "El Mare Nostrum Digital: mito, ideología y realidad de un imaginario sociotécnico". Revista Icono14, 12. Madrid, Asociación Científica de Comunicación y Nuevas Tecnologías ICONO, pp.7-30.

GRANDÍO, María del Mar y BONAUT, Joseba (2012): “Transmedia audiences and television fiction: A comparative approach between Skins (UK) and El Barco (Spain)". Participations. Journal of Audience \& Reception. 9, 2.

HENNIG-THURAU, Thorsten; WIERTZ, Carolina; \& FELDHAUS, Fabian (2012): "Exploring the «Twitter Effect»: An Investigation of the Impact of Microblogging Word of Mouth on Consumers' Early Adoption of New Products". Social Science Research Network. http://ssrn.com/abstract $=2016548$

HERNÁNDEZ PACHECO, José Luis y MIRAFLORES GÓMEZ, Emilio (2012): "SMS: la alfabetización de los jóvenes en el siglo XXI" en La Nueva Alfabetización: un reto para la Educación del Siglo XXI www.cesdonbosco.com/revista/congreso/21-Jose\%20Luis\%20Hernandez\%20Pacheco.pdf. [fecha de consulta: 8 de febrero de 2012].

HIGHFIELD, Tim; HARRINGTON, Stephen \& BRUNS, Axel (2013): Twitter as a Technology for Audiencing and Fandom: The \#Eurovision Phenomenon. Information, Communication \& Society, 16 (3), pp. 315-339. 
JENKINS, Henry (2009): Fans, blogueros y videojuegos. Barcelona, Paidós.

JENSEN, Klaus Bruhn (2009): "Three-Step Flow". Journalism, 10 (3). Pennsylvania, SAGE, pp. 335-337.

LARSSON, Aanders Olof \& MOE, Hallvard (2011): "Studying political microblogging: Twitter users in the 2010 Swedish election campaign". New Media and Society: http://nms.sagepub.com/content/early/2011/11/21/1461444811422894. [fecha de consulta: 8 de febrero de 2012].

EUN-JU Lee \& YUN SHIN, Soo (2012): "When the Medium Is the Message: How Transportability Moderates the Effects of Politicians Twitter Communication". Communication Research published online 23 November 2012. Doi: $10.1177 / 0093650212466407$

MARWICK, Alice E. (2010): "I tweet honestly, I tweet passionately: Twitter users, context collapse, and the imagined audience". New Media \& Society. July 7.

MARWICK, Alice E. \& BOYD, Danah (2011): "To See and to Be Seen: Celebrity practice on Twitter". Convergence: The International Journal of Research into New Media Technologies, 17. Bedforshire, SAGE, pp. 139-157.

MCKINNEY, Bruce; KELLY, Lynne; \& DURAN Robert L. (2012): "Narcissism or Openness?: College Students. Use of Facebook and Twitter". Communication Research Reports. Volume 29, No. 2, April-June 2012, pp. 108-118.

MOROZOV, Evgeny (2009): The Dark Side of Internet Freedom. The Net Delusion. New York, Public Affairs.

OLORUNNISOLA, Anthony \& BRANDIE, Martin (2012): "Influences of media on social movements: Problematizing hyperbolic inferences about impacts". Telematics and Informatics 30 (2013), pp. 275-288. http://dx.doi.org/10.1016/j.tele .2012.02.005.

NÚÑEZ-GÓMEZ, Patricia; GARCÍA-GUARDIA, María Luisa \& HERMIDAAYALA, Lourdes Ainhoa (2012): "Tendencias de las relaciones sociales e interpersonales de los nativos digitales". Revista Latina de Comunicación Social, 67. Tenerife, Universidad de La Laguna, pp. 179-202.

PRENSKY, Mark (2001): "Digital natives, digital inmigrants". On the Horizon 9 (5). Bradford, MCB University Press, pp. 1-6.

PAPACHARISSI, Zizi \& OLIVEIRA, Maria de Fatima (2012): “Affective news and networked publics: the rhythms of news storytelling on \#Egypt". Journal of Communication. Volume 62, pp. 266-282.

PRÁDANOS, Eduardo (2012): “La «televisión social»: ¿tecnología o algo más?”. Genbeta Social Media: www.genbetasocialmedia.com/estrategias-social-media/latelevision-social-tecnologia-o-algo-mas-por-eduardo-pradanos. [fecha de consulta: 8 de febrero de 2012].

PRENSKY, Marc (2001): "Digital Natives, Digital Inmigrants". On the Horizon MCB University Press, 9 (5), pp. 1-6. 
ROJO PIZARRO, Rodrigo (2012): "Existe alguna relación entre la Audiencia Televisiva y la Cantidad de Tweets sobre un programa: El Caso de Mundos Opuestos". Mención Administración. Universidad de Chile. Facultad de Economía y negocios. http://tesis.uchile.cl/handle/2250/111105.

SAID HUNG, Elias y ARCILA CALDERÓN, Carlos (2011): "Los líderes de opinión en Colombia, Venezuela e Irán. El caso de los 20 usuarios más vistos en Twitter". Comunicación y Sociedad, 24 (1). Navarra, Universidad de Navarra, pp.75-100.

SERRANO-PUCHE, Javier (2012): "Herramientas web para la medición de la influencia digital: análisis de Klout y PeerIndex”. El profesional de la información, 21 (3), pp. 298-303: http://dx.doi.org/10.3145/epi.2012.may.11

THORSON, Kjerstin; DRISCOLL, Kevin; EKDALE, Brian; EDGERLY, Stephanie; THOMPSON, Liana Gamber; SCHORK, Andrew; SWARTZ, Lana; VRAGA, Emily; \& WELLS, Chris (2013): "Youtube, Twitter and the occupy movement. Connecting content and circulation practices". Information, Communication \& Society. Volume 16, Issue 3. Routledge. Doi:10.1080/1369118X.2012.756051.

WILSON, James (2011): "Playing with politics: Political fans and Twitter faking in post-broadcast democracy". Convergence. November, vol. 17, no. 4, pp. 445-461 (Doi: 10.1177/1354856511414348)

YEON-OK, Lee \& WOO PARK, Han (2010): "The Reconfiguration of E-Campaign Practices in Korea: A Case Study of The Presidential Primaries of 2007". International Sociology, vol. 25, 1, pp. 29-53.

\section{Apoyos y agradecimientos}

Este trabajo forma parte de un estudio mayor financiado en el ámbito de un Proyecto de Investigación I+D+i. Debemos agradecer también a Mari Luz Congosto (Universidad Carlos III de Madrid) y Tuitele. 